\title{
Annealing study of two-dimensional patterned Ge nanostructures via nanosphere lithography
}

Research Article

\author{
Magdalena Ulmeanu ${ }^{1 *}$, Iuliana lordache ${ }^{1}$, Mihaela Filipescu ${ }^{1}$, Valentin Craciun ${ }^{1,2}$, \\ Simona Cinta Pinzaru ${ }^{3}$ and Andreas Hörner ${ }^{4}$
}

1 National Institute for Laser, Plasma and Radiation Physics, PO Box MG-16 Magurele, 077125 Bucharest, Romania

2 Materials Science and Engineering, University of Florida, Gainesville, FL 32611, USA

3 Physics Department, Babes-Bolyai University, Kogalniceanu 1, RO-3400, Cluj-Napoca, Romania

4 Institute of Physics, Experimentalphysics I, University Augsburg, 86135 Augsburg, Germany

Received 28 October 2010; accepted 10 March 2011

\begin{abstract}
:
Experimental studies on patterning hexagonal Ge nanostructures have been conducted on Si substrates through deposition of $\mathrm{Ge}$ with colloidal particles as a mask. The deposited Ge thin film possesses, according to the X-ray diffraction measurements, in plane texture, being epitaxial and aligned with the (111) $\mathrm{Si}$ substrate. The size distribution of the patterned Ge nanostructures is narrow, as indicated by the atomic force microscopy and scanning electron microscopy measurements. We have obtained Ge nanostructures with lateral dimension of $490 \mathrm{~nm}$ (height $12 \mathrm{~nm}$ ), $200 \mathrm{~nm}$ (height $6 \mathrm{~nm}$ ) and $82 \mathrm{~nm}$ (height $6 \mathrm{~nm}$ ) by using different sizes of polystyrene spheres. We have performed in depth studies of the Ge nanostructures' behavior due to thermal and rapid thermal post-annealing processes. FT micro-Raman spectroscopy shows that there is no Si intermixing during the annealing process. In order to quantify the changes in the height and lateral dimension, we have performed atomic force microscopy and white light interferometry analysis. The changes in shape and the decrease in the area of a cross-section of Ge nanostructure will be discussed in respect to similar results shown in the literature for Ge thin films during the annealing process.
\end{abstract}

PACS (2008): 81.07.-b, 81.16.-c, 81.40.-z, 81.70.-q

Keywords: $\quad$ Ge nanostructure $\cdot$ colloidal lithography $\cdot$ thermal annealing

(C) Versita Sp. z o.o.

\section{Introduction}


the fundamental research concerning the understanding of growth processes $[2,3]$. In the quest to expand integrated silicon technology, in particular to applications in optoelectronics, Ge/Si nanoheterostructures [4] with engineered band structures have come under intense investigation as important candidates for light-emitting quantum dot (QD) based devices.

Research efforts have been dedicated to the exploration of "dotlike" structures obtained via the Stranski-Krastanov (SK) growth mode, which comprises the formation of a wetting layer (WL) followed by three-dimensional island ("dot") formation that relaxes the strain induced by the $4.2 \%$ lattice mismatch between $\mathrm{Ge}$ and $\mathrm{Si}$. To control the size, shape and density, but mostly the spatial positioning of Ge dots, many strategies including combinations of lithography-based (top down) and spontaneous self-organization approaches (bottom up) have been pursued [5-8]. Work has focused on the assisted organization of $\mathrm{Ge}$ dots grown on prepatterned $\mathrm{Si}$ or $\mathrm{SiO}_{2}$ substrates either by chemical vapor deposition (CVD) [9, 10], molecular beam epitaxy (MBE) [11-13], or electron-beam evaporation with nanosphere lithography [14].

In this paper, we have also used the method of patterning Ge nanostructures via electron-beam evaporation using a colloidal particles mask. The intent of this approach is two-fold: first, to investigate the kinetic process of electron beam evaporation of Ge thin film and Ge nanostructures on a Si substrate by electron beam-evaporation; and second to make in depth analysis of the thermal treatments on Ge nanostructures with the purpose to further decrease their size by future studies on isotropic oxidation processes [15]. First we have characterized the Ge thin films by collecting grazing incidence and symmetrical $X$-ray diffraction patterns from the film (GIXD and XRD) and $X$-ray reflectometry (XRR). Before and after annealing, the resulting $\mathrm{Ge}$ nanostructures were visualized by Atomic Force Microscopy (AFM) and Scanning Electron Microscopy (SEM). Previous thermal annealing studies have been performed in the literature on Ge nanostructures [14]. We have varied the size of the Ge nanostructures and the annealing methods, i.e. thermal annealing and fast thermal annealing in order to get a deeper and more quantitative analysis of the results of the annealing treatment of Ge nanostructures. In our case the Ge nanostructure showed similar behavior with the annealing effects to that observed in the case of Ge thin film, i.e. a loss of material due to the oxidation and thermal desorption of the $\mathrm{Ge}$ oxides, which take place simultaneously [16]. In order to make statistical measurements we have employed, along with the AFM technique, the White Light Interferometry (WLI) technique. In this paper, we report for the first time, that WLI should also be used with caution when it is used to measure the profile of triangular pyramid Ge nanostructures. The comparison and the results of both techniques will be discussed for three different sizes of Ge nanostructures.

\section{Experimental methods}

Initially, a monolayer of colloidal particles is selfassembled on a Si (111) substrate (purchased from Crystal $\mathrm{GmbH}$ ) in a hexagonally close-packed arrangement [17]. Although areas without self-assembled nanospheres were observed throughout the surface, domains of closepacked monolayers of the polystyrene (PS) nanospheres extended over several hundred square micrometers and free of defects were observed by microscopy images. A metallic material is deposited onto the surface through the interstices of a colloidal template. The lateral dimensions of the nanopatterned features are determined by the interstitial spacings between the close-packed colloidal particles, while the height is controlled by the amount of material deposited [18]. Ge (99.999\%) purchased from Umicore was used for deposition to form the nanostructure. The material was evaporated from an e-beam source vacuum evaporator (BOC Edwards Auto 500) with a base pressure of $10^{-9}$ Torr, while the substrate was kept at room temperature. A microbalance was used to monitor the evaporation rate and the thickness of the Ge thin film, with the microbalance calibrated by profilometry measurements. The deposition rate was $\sim 0.1 \mathrm{~nm} / \mathrm{min}$ at a working pressure of $5 * 10^{-6}$ Torr. The PS monolayer mask was mechanically lifted off by using a tape or by ultra-sonication with toluene or clorofom. After reloading the samples into a home-made furnace or a rapid thermal annealing (RTA) source heating (purchased from ULVAC-RIKO), sample annealing were conducted and the temperature was monitored by an automatic temperature controller. For each colloidal particle mask, we have prepared three different samples. One sample was not-annealed and the other two were annealed and analyzed. For the homemade furnace the heating rate was $400^{\circ} \mathrm{C} /$ hour, the time of annealing, after reaching the desired annealing temperature, was 45 minutes, and the annealing temperature $\mathrm{T}_{\text {anneal }}$ was from $600^{\circ} \mathrm{C}$ to $925^{\circ} \mathrm{C}$. For the RTA, the heating rate was $50^{\circ} \mathrm{C} / \mathrm{sec}$, the time of annealing 30 minutes, and $\mathrm{T}_{\text {annel }}=925^{\circ} \mathrm{C}$, close to the melting temperature of the Ge.

X-ray reflectivity (XXR) curves were collected from the deposited Ge film with the aid of an X'Pert MRD instrument having a mirror and a $(1 / 32)^{\circ}$ slit in the primary optics, and a thin film collimator, a $0.1 \mathrm{~mm}$ slit and a graphite monochromator on the secondary optics. The divergence 
of the parallel beam used in these experiments was $0.008^{\circ}$. The same instrument was used to collect grazing incidence and symmetrical X-ray diffraction patterns from the film (GIXD and XRD). For these measurements, the $0.1 \mathrm{~mm}$ slit was removed while the $(1 / 32)^{\circ}$ slit was replaced with a $(1 / 8)^{\circ}$ slit for GIXD, or with a $(1 / 2)^{\circ}$ slit for XRD, respectively. For the AFM analysis, we have used a piece of equipment from Park Instruments, with a piezo scanner with a maximum image scan size of $50 \mu \mathrm{m}^{2}$. The WLI experiments were carried out with a Mirau interference microscope (Ambios $\mathrm{Xi}-100)$ and a $10 \times$ or $50 \times$ magnification objective was used. Correlograms were detected by a CCD video camera. A piezoelectric transducer was used for the vertical scanning. A broadband light source with a center wavelength of $550 \mathrm{~nm}$ is used.

\section{Results and discussion}

\subsection{Structural characterization of the Ge thin films}

To asses the out of plane and in plane texture of the Ge films, $\omega$-rocking curves and phi-scans were collected using a $(1 / 2)^{\circ}$ slit with a mirror and a four bounce Ge monochromator in the primary optics and a $1 \mathrm{~mm}$ brass slit in front of the detector in the secondary optics. In Figure 1 two typical XRR curves collected from the Ge film deposited on $\mathrm{Si}$ are displayed. The results of the simulations of these curves using the Wingixa software program from Panalytical provided a density value of the Ge film from 4.44 to $4.48 \mathrm{~g} / \mathrm{cm}^{3}$, significantly lower than the tabulated density of Ge of $5.35 \mathrm{~g} / \mathrm{cm}^{3}$. These results indicate that the deposited film could contain voids or other defects. The thickness of the film, estimated from the oscillations of the

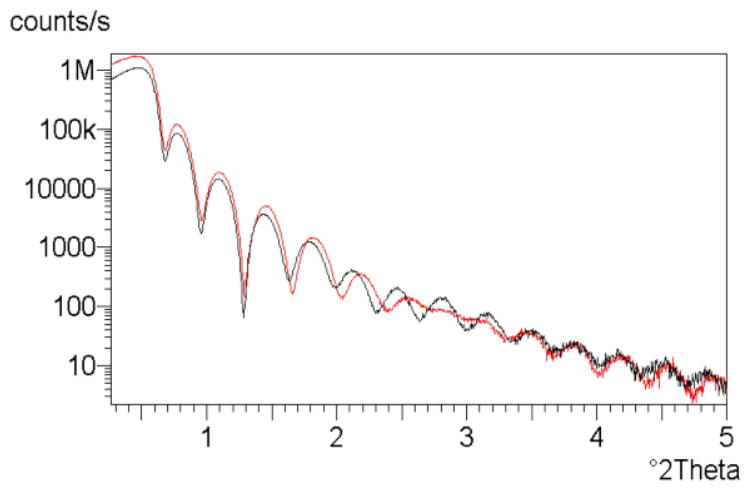

Figure 1. Two XRR curves measured in different locations on the $\mathrm{Ge} / \mathrm{Si}$ sample.
XRR curves, was around $12 \mathrm{~nm}$, while the surface roughness (rms value) was found to be around $0.3-0.4 \mathrm{~nm}$, indicative of a rather smooth surface. Figure 2 shows the diffraction patterns acquired at grazing and symmetrical incidence (denoted GIXD and XRD, respectively, in Figure 2). One could note that the Ge film exhibited only the (111) diffraction line, indicating the deposition of a highly textured film, with the [111] axis perpendicular to the substrate. Two small bumps present in the XRD pattern were tentatively assigned to $\mathrm{GeO}_{2}$ phases. Since there were no such diffraction peaks present in the GIXD pattern we believe that the oxide might also be textured. $\omega$-rocking curves and phi-scans showed that the deposited Ge film also possesses in plane texture, being epitaxial and aligned with the (111) Si substrate.

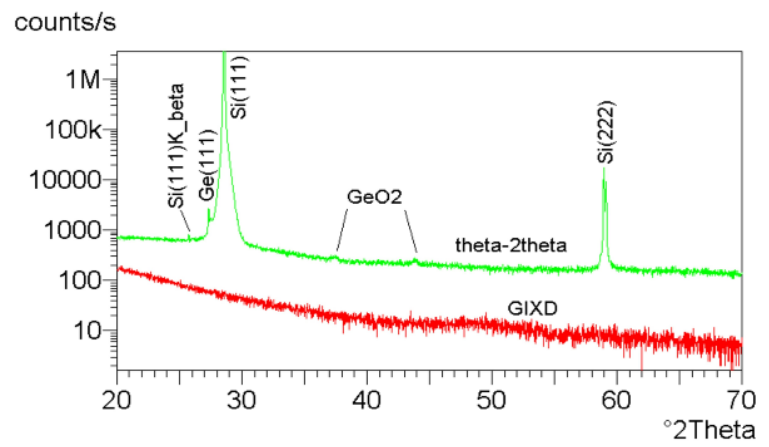

Figure 2. X-ray diffraction patterns acquired from the Ge film.

\subsection{Ge nanostructures}

When equally-sized spherical particles are closely packed on a surface in a single layer, the spheres form a periodical hexagonal pattern. With the monolayer as a mask, the deposited pattern due to the Ge flax passing through the space between the spheres will also form a hexagonal pattern. In our experiment, such patterning was achieved in the Ge/Si system, where a monolayer of polystyrene nanospheres (latex particles) was used as deposition mask. A typical nanosphere lithography (NSL) fabrication is shown in the SEM images in Figure 3, where an ordered hexagonal pattern of Ge nano-structures is obtained with $12 \mathrm{~nm}$ of Ge deposition on the Si surface that was masked with $1500 \mathrm{~nm}$ latex nanospheres (3a), and $6 \mathrm{~nm}$ of Ge deposition on the Si surface with $300 \mathrm{~nm}$ latex nanospheres (3b). A cluster-size distribution measurement from the AFM image reveals that the size dispersion of the Ge nanostructures in the pattern is very narrow, with a peak width at the half maximum (FWHM) that is less than $10 \%$ with respect to the measured size peak. 

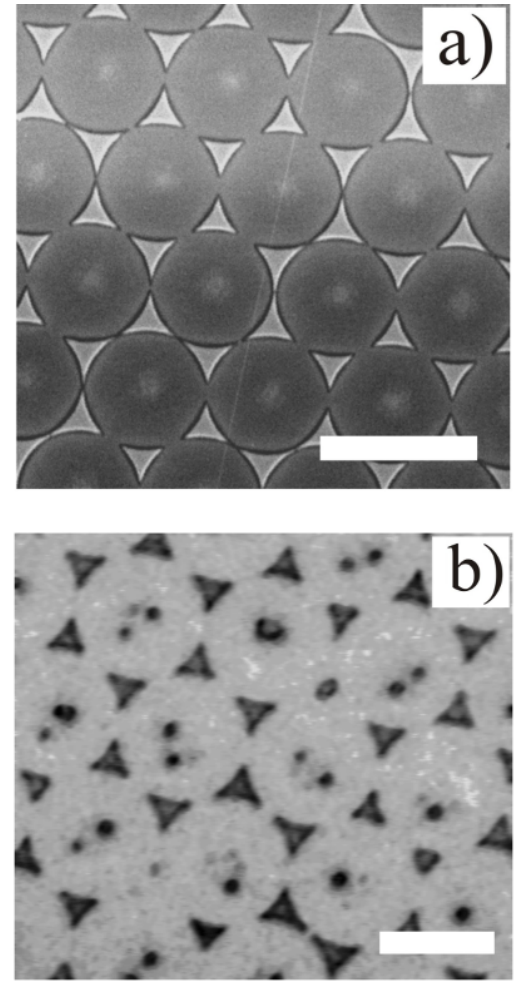

Figure 3. SEM images of the Ge nanostructures obtained for a) colloidal particle mask of $1500 \mathrm{~nm}$ diameter (scale bar $1500 \mathrm{~nm})$ and b) colloidal particle mask of $300 \mathrm{~nm}$ diameter (scale bar $300 \mathrm{~nm}$ ).

We note that the small black dots in between the regular Ge nanostructures (Figure $3 b$ ) are due to the impurities that are present in the colloidal particle suspension. This demonstrates that the NSL is a technique capable of fabricating ordered arrays of semiconductor nanostructures with good size control. We have used colloidal particles with different diameters $D$. The height and the lateral dimension of the Ge nanostructures obtained are listed in Table 1. Table 1 includes different error bars, like the ones for the standard deviation of the diameter of the colloidal particles (purchased from Polyscience) and the AFM measurements of the height and lateral length of the

Table 1. The height and the lateral dimension of the Ge nanostructures obtained after the removal of the colloidal particles mask.

\begin{tabular}{ccc}
\hline $\begin{array}{ccc}\text { Colloidal particle diameter } \\
D[\mathrm{~nm}]\end{array}$ & Height $[\mathrm{nm}]$ & Lateral $[\mathrm{nm}]$ \\
\hline \hline $1500 \pm 39$ & $12 \pm 0.5$ & $490 \pm 2$ \\
$700 \pm 25$ & $46 \pm 0.5$ & $200 \pm 2$ \\
$300 \pm 14$ & $6 \pm 0.5$ & $82 \pm 2$ \\
\hline
\end{tabular}

Ge nanostructures. As previously mentioned, the height is dependent on the amount of material evaporated, while the lateral dimension is given by the diameter of the colloidal particles forming the lithographic mask.

\subsection{Annealing of the Ge nanostructures}

As previously mentioned in the introduction, the annealing behavior of Ge thin film native oxides [16] and Ge nanostructures fabricated with the NSL technique has been studied in the literature [14]. It was found that the native oxide state naturally formed in the air was found to be primarily $\mathrm{GeO}_{2}$ with small amounts of $\mathrm{GeO}_{x}(x<2)$. At $500^{\circ} \mathrm{C}$, most of the $\mathrm{Ge}$ oxides were desorbed through an interfacial reaction of $\mathrm{GeO}_{2}+\mathrm{Ge}+2 \mathrm{GeO}$ between the native oxide layer and the Ge substrate. This is then followed by desorption of volatile $\mathrm{GeO}$ from the surface, with it being reported in the literature that $\mathrm{GeO}$ sublimates at low temperature $[19,20]$. Secondary ion mass spectroscopy (SIMS) profiles confirm the loss of $100 \mathrm{~nm}$ from the Ge layer at $600^{\circ} \mathrm{C}$. X-ray photoelectron spectroscopy (XPS) analysis indicates that this proceeds by oxidation and thermal desorption of the Ge oxides, which take place simultaneously [16]. The shape variation of the Ge nanostructures through thermal annealing resulted, in the above mentioned reference [14], in a change of their dimensions while their volume remained constant. In the particular case of the triangular Ge nanostructure with an in-plane dimension of $\sim 70 \mathrm{~nm}$ and a height of $6 \mathrm{~nm}$, the shape change results in a nanosphere of $\sim 30 \mathrm{~nm}$ in diameter, a true three-dimensional dot. Such a size-variation against volume-conservation phenomenon is interesting since it suggests a new possibility for size-modulation of the Ge nanostructures. We have also performed thermal annealing procedures for the Ge nanostructures, with the sizes listed in Table 1. In our case, the lateral dimension size is considerably bigger than the height. Prior to the annealing treatments, we kept the Ge nanostructures in air conditions, that is why we will consider them natively oxidized. The annealing was made either in a home-made heat pipe inside a furnace to achieve isothermal conditions over the whole length of the sample or by rapid thermal annealing in a commercial made system. In the first case, to prevent further oxidation effects, we have used a $90 \% \mathrm{~N}_{2}+10 \% \mathrm{H}_{2}$ gas flow atmosphere (heating rate $400^{\circ} \mathrm{C} /$ hour, time of annealing, after reaching the desired annealing temperature, was 45 minutes, annealing temperature $\mathrm{T}_{\text {anneal }}$ was from $600^{\circ} \mathrm{C}$ to $925^{\circ} \mathrm{C}$ ), and in the second case in a vacuum (heating rate $50^{\circ} \mathrm{C} / \mathrm{sec}$, time of annealing 30 minutes, $\mathrm{T}_{\text {annel }}=925^{\circ} \mathrm{C}$, close to the melting temperature of the Ge).

In order to quantify the shape variation of the Ge nanos- 
tructures due to the annealing procedures, we have employed two different techniques: AFM and WLI. AFM will provide information about the lateral and height dimension of the Ge nanostructures. Although AFM is a highresolution technique [21], it takes a lot of time to perform statistic measurements on different areas of the samples. WLI is a recent technique used to assess surface topography. This non-contact method quickly measures the 3D geometry of surfaces. Based on the reflection from the measured surface of a white light beam into an interferometric objective, this technique provides quantitative information for much larger areas (up to some square millimeters) with a vertical resolution around ten nanometers. Thus, WLI is another technique that can be used for height measurement in the vertical direction, along with complete morphological and analytical data from AFM. We consider that this kind of study is important to be made when evaluating the effects of annealing on the Ge nanostructures.

In Figure 4, we have represented WLI processed surface profiles of: i) a standard step-height (Ambios, height $1.2 \mu \mathrm{m})$, ii) a step height between an empty area and the group of closely compact area of colloidal particles with the diameter of $3 \mu \mathrm{m}$, respectively diameter of $1.5 \mu \mathrm{m}$, iii) a step height profile between a compact area with $\mathrm{Ge}$ nanostructures (Area 2 in Figure 4iii) and an area free of Ge nanostructures, i.e. Si substrate (Area 1 in Figure 4iii). The closely compact areas, i.e. of colloidal particles and Ge nanostructures, are detected by the WLI as single areas with a step height equal to the diameter of the colloidal particles, or with the height of the Ge nanostructures, respectively. If the colloidal particles are not self-assembled in closely compact areas or the area counts only a few colloidal particles it is possible to detect by WLI measurements the profile of the hole between the colloidal particles (with the hole obviously equal to the diameter of the colloidal particles). The advantage of the WLI is that it will allow us to make quick statistical measurements on the Ge nanostructures by investigating different areas on the sample. This type of measurement will complete the AFM information.

The shape variation of the Ge nanostructures made using a mask of colloidal particles $(D=1500 \mathrm{~nm})$ through thermal annealing is shown in Figure 5a. Figure 5b presents the AFM image of one Ge nanostructure. As can be seen in Figure $5 c$, the shape of the Ge nanostructures shows obvious height and less obvious lateral dimension modifications. Concerning the height modifications, we can observe that the two height peaks of the annealed Ge nanostructure are equal in size with the height peak of the Ge nanostructures that were not annealed, indicating a loss of material in the central part of the Ge nanostructure. The calculated area of a cross-section at a height
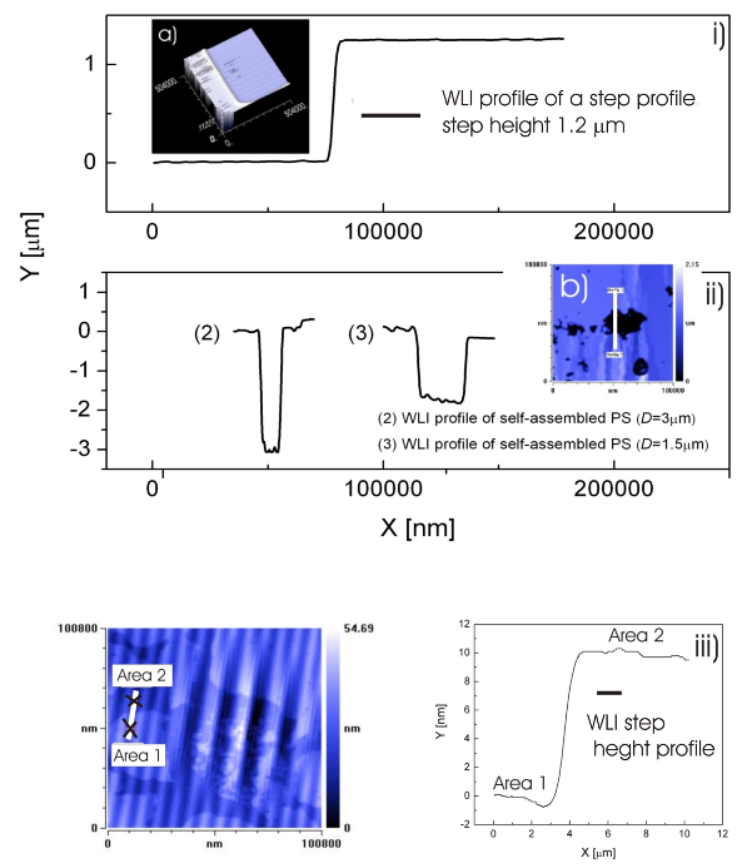

Figure 4. WLI profiles of: i) a standard step profile, height $1.2 \mu \mathrm{m}$, 3D image of the profile in the image a), ii) self-assembled colloidal particles $(D=3 \mu \mathrm{m})$, self-assembled colloidal particles $(D=1.5 \mu \mathrm{m})$, iii) Ge nanostructures, optical microscopy of the Ge nanostructures (Area 2) on a Si substrate (Area 1).

equal to the apex of the Ge nanostructure, according to the AFM scan lines represented in Figure $5 c$, indicates a value of $205 \mathrm{~nm}^{2}$ for the Ge not-annealed nanostructure and $165 \mathrm{~nm}^{2}$ for the Ge annealed nanostructure. By varying the time of annealing, the annealing temperature in the interval $600-925^{\circ} \mathrm{C}$ or the annealing procedures: thermal or rapid annealing, we did not observe further shape changes in the Ge nanostructure. We assume that the loss of material is similar to that observed with the annealing treatments for Ge thin films and Ge nanocrystals in $\mathrm{SiO}_{2}$ films [22]. At this point we do not have an explanation for the preferential central area of the Ge removal inside the Ge nanostructure represented in Figure 5c. Further analysis should provide useful information about the chemical map of the nanostructure.

For the case of the not-annealed sample, the WLI statistical results indicate a height of $15 \mathrm{~nm}$ for the Ge nanostructures, compared with the AFM evaluation of $12 \mathrm{~nm}$. For the case of the annealed sample, the WLI statistical measurements indicate a height profile of $23 \mathrm{~nm}$, compared with the highest peak of the AFM profile of $12 \mathrm{~nm}$. The AFM and WLI measurements coincide, in the limit of the error bar for each measurement, for the not-annealed case. For the annealed case, there appears to be significant dif- 


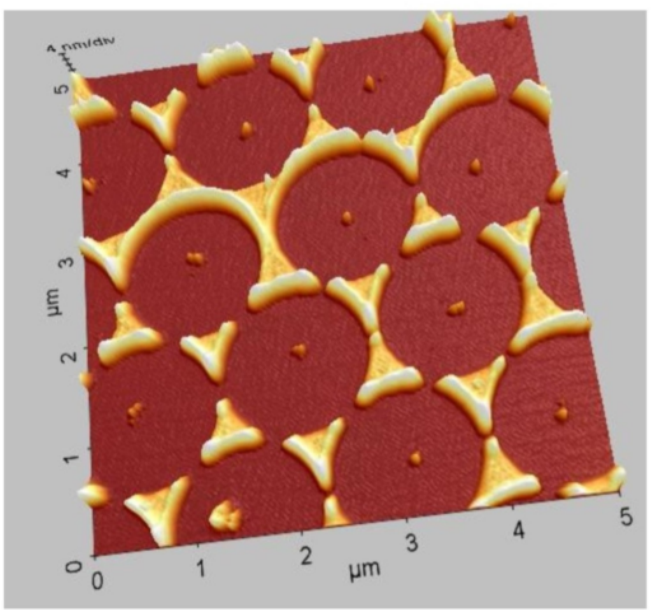

a
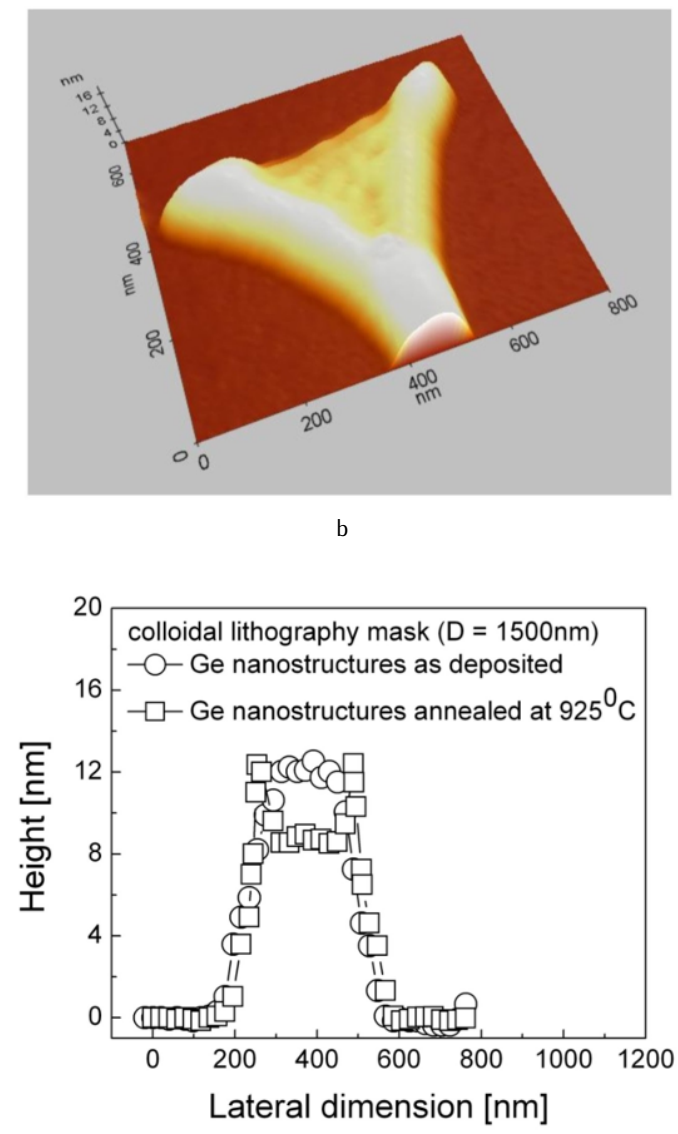

Figure 5. AFM image of: a) Ge nanostructures obtained with a colloidal particles mask of $D=1500 \mathrm{~nm}$ after the annealing treatments, b) single Ge nanostructure obtained with a colloidal particles mask of $D=1500 \mathrm{~nm}$ after the annealing treatments, c) AFM scan line comparison between the Ge nanostructure before and after the annealing treatments. ferences in the evaluation of the height after the annealing treatment, with the height evaluation of the WLI being almost 2 times larger when compared with the AFM. We assume that the differences in the WLI and AFM results for the annealed samples may be induced by a change in the reflectivity of the Ge nanostructures due to the annealing procedures.

In the case where the Ge nanostructures are obtained with a colloidal lithography mask of colloidal particles with $D=700 \mathrm{~nm}$, we observe Ge annealed nanostructures with a more rounded shape, as evident in the AFM images shown in Figure 6a. To quantify the change in the area, we have represented in Figure $6 \mathrm{~b}$ the area of the Ge nanostructure, as calculated according to the AFM scan lines, at different temperature values, starting from
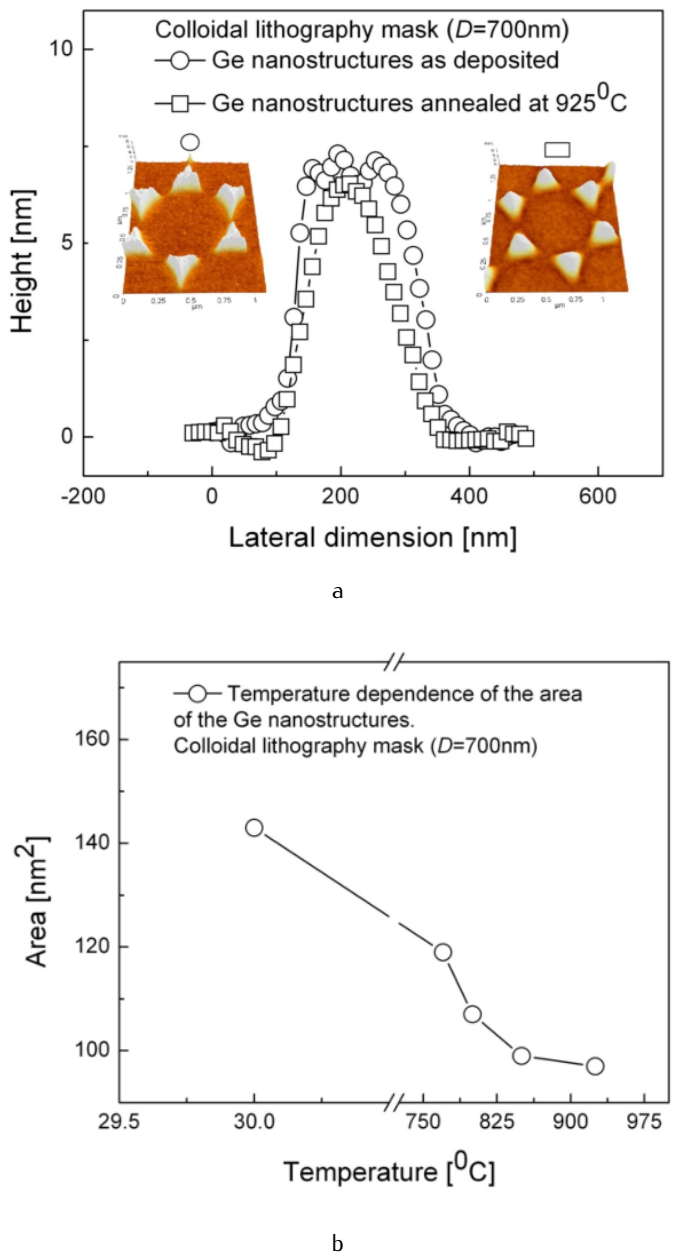

Figure 6. a) AFM scan line comparison between the Ge nanostructure obtained with a colloidal particles mask of $D=$ $700 \mathrm{~nm}$ before and after the annealing treatments, b) temperature dependence of the area of a cross-section of the Ge nanostructure. 
room temperature. The graphic shows a decrease of $30 \%$ of the area of a cross-section of the Ge nanostructure at the final temperature of $925^{\circ} \mathrm{C}$. As in the previous case, the WLI measurements provided evidence of an increase of $57 \%$ of the sample height for the annealed Ge nanostructure. This type of height increase was not evident in the AFM measurements.

In Figure 7 we present Ge nanostructures, after the annealing process, made with a mask of colloidal particles with $D=300 \mathrm{~nm}$. As can be remarked from Figure 7b, almost no shape changes can be detected from the scan lines in the AFM images. The result is independent of the annealing time temperature or temperature range up to $925^{\circ} \mathrm{C}$. The shape change becomes even more complicated to evaluate by the WLI analysis. As shown in the SEM image (Figure 8), there are large areas of colloidal

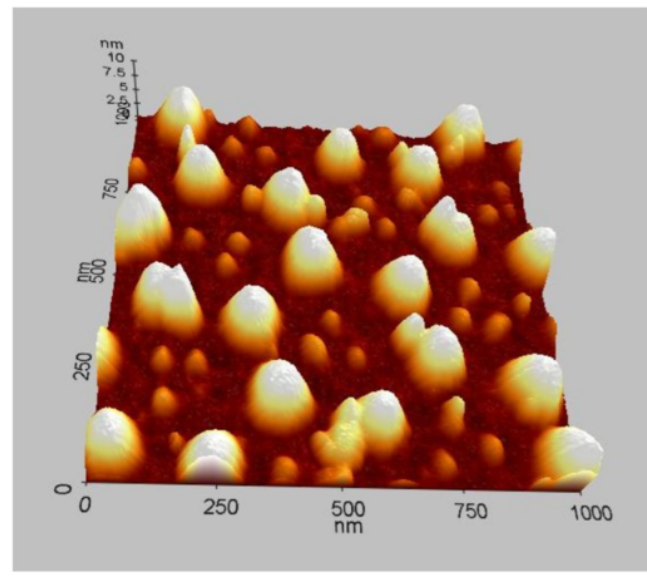

a

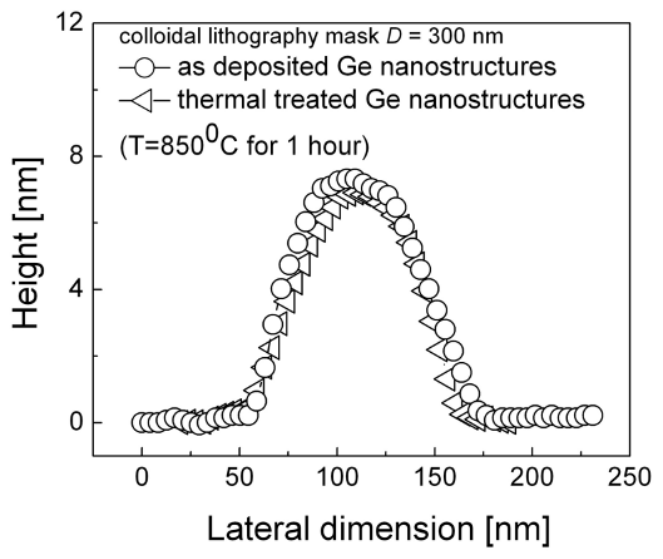

Figure 7. AFM image of: a) Ge nanostructures obtained with a colloidal particles mask of $D=300 \mathrm{~nm}$ after the annealing treatments, b) AFM scan line comparison between the $\mathrm{Ge}$ nanostructure before and after the annealing treatments. particles not removed by the tape. Even when using chemicals, islands with colloidal particles are still present. The evaluation by WLI of a step profile for such areas is difficult even when using a $50 \times$ objective microscope.

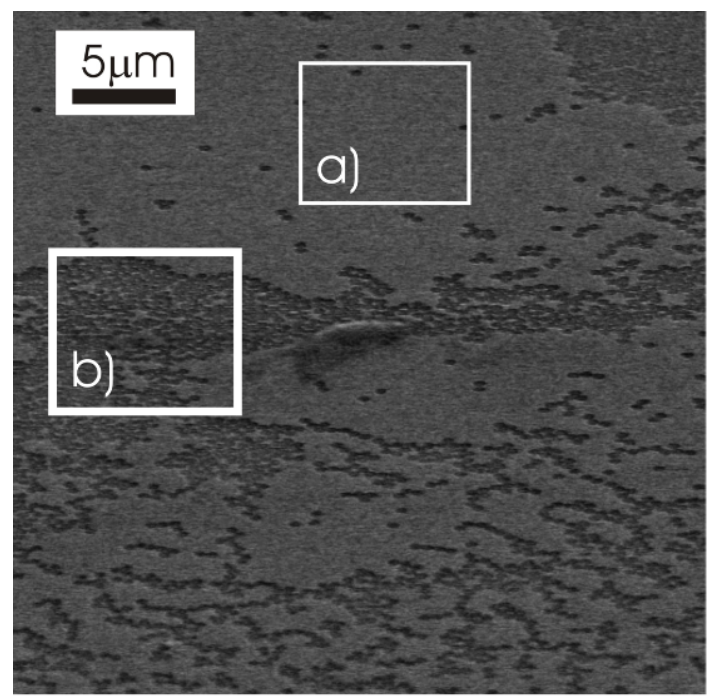

Figure 8. SEM image of an area covered with: a) colloidal particles and b) Ge nanostructures.

Fourier Transform (FT)-Raman spectra were recorded using an Equinox 55 Bruker spectrometer with an integrated FRA-106 S Raman module fiber optic coupled to the Ramanscope II micro-Raman equipment. A Nd:YAG laser operating at the $1064 \mathrm{~nm}$ line was employed for excitation. The laser power was $350 \mathrm{~mW}$ and 200 scans were collected for each spectrum represented in Figure 9. The detection of the Raman signal was carried out with a ni-

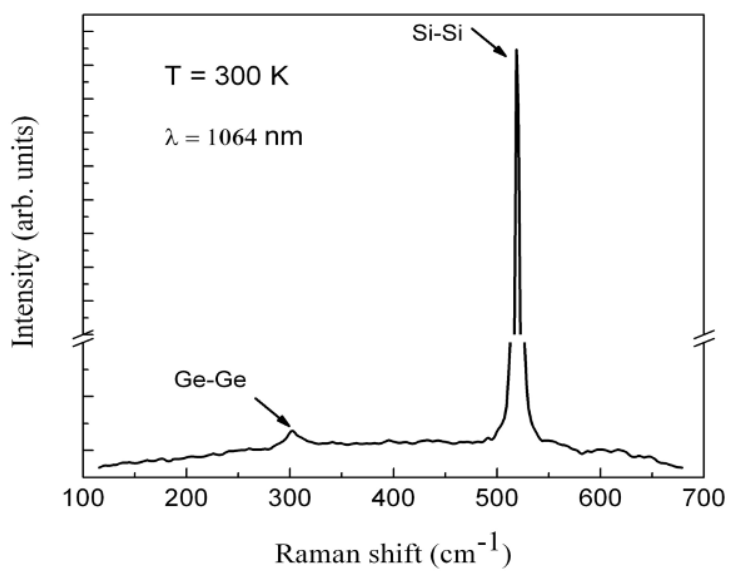

Figure 9. FT micro-Raman spectra acquired by probing the Ge nanopatterned areas after the annealing procedures. 
trogen cooled $\mathrm{Ge}$ detector. The spectral resolution was $4 \mathrm{~cm}^{-1}$. In all the micro-Raman spectra the dominant band at $520 \mathrm{~cm}^{-1}$ characteristic for the Si substrate keeps its position. Additionally, a very weak band at $302 \mathrm{~cm}^{-1}$ is observed for the sample in the annealed case, which corresponds to the measured optical phonon mode of the crystalline Ge reference. The absence of the Si-Ge phonon band around $\sim 400 \mathrm{~cm}^{-1}$ clearly indicates that there is no Si-Ge intermixing during the annealing process.

\section{Conclusion}

Ge nanostructures, with lateral sizes from 150 to $400 \mathrm{~nm}$ and height from 6 to $12 \mathrm{~nm}$, have been obtained using the combination of NSL and e-beam evaporation. The 2D ordered structures on Si (111) substrates are evident in SEM and AFM images. The changes in the height and lateral dimensions of the Ge nanostructures due to annealing treatments have been analyzed by AFM and WLI measurements. In our case, we did not observe, as previously shown in the literature, volume conservation due to the thermal annealing procedures, but a behavior more similar to the native oxides Ge thin films where a loss of Ge was observed in the annealing procedures. The WLI measurements provide evidence of changes in the height of $\mathrm{Ge}$ nanostructures due to annealing that are almost twice that of the AFM measurements. For the case of not-annealed samples, the WLI and AFM measurements indicate similar results, in the limit of the error bars. We assume that the differences in the WLI and AFM results for the annealed samples may be induced by a change in the reflectivity of the Ge nanostructures due to the annealing procedures. The FT Raman spectroscopic measurements show that the Ge nanostructures under study are crystalline after the thermal annealing procedures.

\section{Acknowledgements}

This work was supported by CNCSIS-UEFISCSU, project PNII-IDEI code 596/2008.

\section{References}

[1] J. Konle, H. Presting, H. Kibbel, R. Sauer, Solid State Electron. 45, 1921 (2001)

[2] G. Medeiros-Ribeiro et al., Science 279, 353 (1998)

[3] J. Stangl, V. Holy, G. Bauer, Rev. Mod. Phys. 76, 725 (2004)

[4] J.M. Baribeau, X. Wu, N.L. Rowell, D.J. Lockwood, J. Phys.-Condens. Mat. 18, R139 (2006)

[5] T.I. Kamins, R.S. Williams, Appl. Phys. Lett. 71, 1201 (1997)

[6] G. Capellini, M. de Seta, C. Spinella, F. Evangelisti, Appl. Phys. Lett. 82, 1772 (2003)

[7] A. Karmous et al., Appl. Phys. Lett. 85, 6401 (2004)

[8] A. Bernardi et al., Appl. Phys. Lett. 89, 101921 (2006)

[9] T.I. Kamins et al., Appl. Phys. Lett. 74, 1773 (1999)

[10] L. Vescan, Mat. Sci. Eng. A-Struct. 302, 6 (2001)

[11] O.G. Schmidt et al., Appl. Phys. Lett. 77, 4139 (2000)

[12] G. Jin, J.L. Liu, K.L. Wang, Appl. Phys. Lett. 76, 3591 (2000)

[13] Z. Zhong et al., J. Appl. Phys. 93, 6258 (2003)

[14] N. Li, M. Zinke-Allmang, Jpn. J. Appl. Phys. 41, 4626 (2002)

[15] V. Craciun, E.S. Lambers, R.K. Singh, I.W. Boyd, Appl. Surf. Sci. 186, 237 (2002)

[16] J. Oh, J.C. Campbell, Appl. Surf. Sci. 33, 364 (2004)

[17] M. Ulmeanu, M. Filipescu, R.V. Medianu, Phys. Status Solidi C 12, 3584 (2008)

[18] F. Burmeister et al., Langmuir 13, 2983 (1997)

[19] K. Prabhakaran, F. Maeda, Y. Watanabe, T. Ogino, Appl. Phys. Lett. 76, 2244 (2000)

[20] D.A. Hansen, J.B. Hudson, Surf. Sci. 292, 17 (1993)

[21] M. Ulmeanu et al., Appl. Surf. Sci. 165, 109 (2000)

[22] V. Beyer, J. von Borany, Phys. Rev. B 77, 014107 (2008) 